\title{
Magnification endoscopy in combination with acetic acid enhancement and narrow-band imaging for the accurate diagnosis of colonic neoplasms
}

\section{(ㄷ)(i)}

Authors

Kotaro Shibagaki ${ }^{1}$, Norihisa Ishimura², Takafumi Yuki ${ }^{3}$, Hideaki Taniguchi ${ }^{4}$, Masahito Aimi ${ }^{4}$, Keita Kobayashi ${ }^{5}$, Satoshi Kotani $^{2}$, Tomotaka Yazaki², Noritsugu Yamashita², Yuji Tamagawa², Tsuyoshi Mishiro² ${ }^{2}$, Shunji Ishihara², Akira Yasuda $^{6}$, Yoshikazu Kinshita ${ }^{2}$

Institutions

1 Department of Endoscopy, Faculty of Medicine, Shimane University, Izumo, Japan

2 Department of Gastroenterology, Faculty of Medicine, Shimane University, Izumo, Japan

3 Division of Gastroenterology, Matsue Red Cross Hospital, Matsue, Japan

4 Department of Gastroenterology, Tottori Municipal Hospital, Tottori, Japan

5 Department of Pathology, Tottori Municipal Hospital, Tottori, Japan

6 Department of Medical informatics, Faculty of Medicine, Shimane University, Izumo, Japan

submitted 6.7.2019

accepted after revision 25.10.2019

Bibliography

DOI https://doi.org/10.1055/a-1068-2056 |

Endoscopy International Open 2020; 08: E488-E497

(c) Georg Thieme Verlag KG Stuttgart · New York elSSN 2196-9736

Corresponding author Kotaro Shibagaki, MD, PhD, Department of Gastroenterology, Faculty of Medicine, Shimane University, 89-1 Enya, Izumo, Japan 693-8501

Fax: +81-85320-2187

kotaro@med.shimane-u.ac.jp

\section{ABSTRACT}

Background and study aims Magnification endoscopy with narrow-band imaging (NBIME) and NBIME with acetic acid enhancement (A-NBIME) enable visualization of the vascular and microstructural patterns of colorectal polyp. We compared the diagnostic accuracy and reproducibility of white light endoscopy (WLE), NBIME, and A-NBIME for predictive histologic diagnosis.

Patients and methods Consecutive colorectal polyps ( $\mathrm{N}=$ 628; 38 hyperplasias, 488 adenomas, 72 M-SM1 cancers, and 30 SM2 cancers) were photographed with WLE, NBIME, and A-NBIME. Endoscopic images were independently reviewed by three experts, according to the traditional criteria for WLE, the Japan NBI Expert Team classification for NBIME, and pit pattern classification for A-NBIME to compare diagnostic accuracy and interobserver diagnostic agreement among modalities.

Results The specificity (95\% confidence interval) of hyperplasia and SM2 cancer with WLE were $98.2 \%$ (96.8\%-99.1\%) and $99.4 \%$ (98.5\%-99.9\%), respectively, showing high accuracy for endoscopic resection without magnifying observation. Diagnostic accuracy of WLE, NBIME, and A-NBIME was $80.8 \%(77.4 \%-83.8 \%), 79.3 \%(75.9 \%-82.4 \%)$, and $86.1 \%(83.2 \%-88.7 \%)$, respectively, showing the highest accuracy for A-NBIME among modalities $(P<.05)$. NBIME showed a lower PPV for M-SM1 cancer $(P<.05)$, as with WLE $(P=.08)$ compared to A-NBIME. Fleiss's kappa values for WLE, NBIME, and A-NBIME diagnosis were 0.43 (0.39$0.46), 0.52(0.49-0.56)$ and $0.65(0.62-0.69)$, respectively, showing insufficient reproducibility of WLE and superiority of A-NBIME among modalities.

Conclusion WLE showed high accuracy for endoscopic resection of colorectal polyps in expert diagnosis. NBIME demonstrated a higher diagnostic reproducibility than WLE. A-NBIME showed possible superiority among modalities in both diagnostic accuracy and reproducibility. 

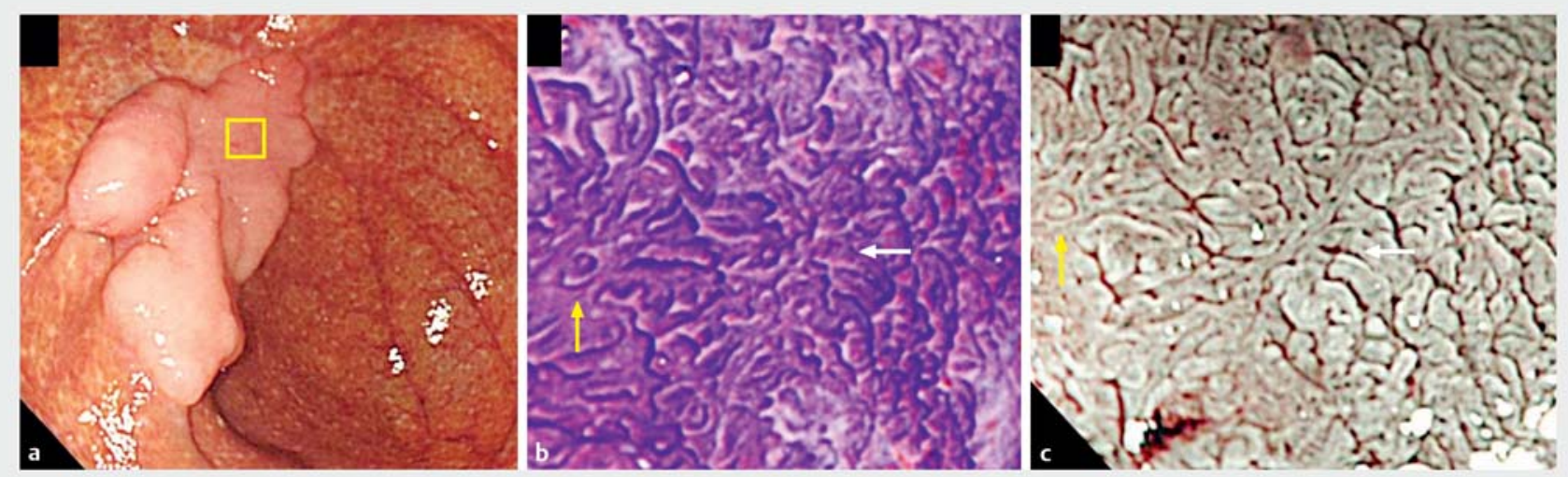

- Fig. 1 a Pit pattern visualized by CV-MCE and A-NBIME. WLE image shows a laterally spreading tumor that was histologically diagnosed as M-SM1 cancer. The opening of glandular crypts is irregularly shaped, but its contour is equivalently clearly visualized between $\mathbf{b}$ CV-MCE and c A-NBIME; yellowish and whitish arrows indicate the same part of the tumor. The pit pattern is diagnosed as type VI-L with both modalities.

\section{Introduction}

Colorectal cancer was the third most commonly diagnosed cancer globally in 2018 [1]. Endoscopic resection of colorectal polyps reportedly contributes to the prophylaxis and improved prognosis of colorectal cancer [2]. However, not all colorectal polyps are candidates for endoscopic resection, and a qualitative endoscopic diagnosis is essential before treatment.

Conventional white-light endoscopy (WLE) is a fundamental modality, but magnification endoscopy is not necessarily globally used due to a requirement for technical expertise. Magnification chromoendoscopy with crystal violet staining (CV-MCE) enables visualization of the pit pattern (the shape of the glandular crypt opening) [3-5], and magnification endoscopy with narrow-band imaging (NBIME) enables visualization of the vessel pattern without mucosal staining [6-8]. Generally, magnification endoscopy is considered to be superior to WLE for the qualitative diagnosis of colorectal polyps, but it has not been investigated fully. In addition, NBIME may be slightly inferior to CV-MCE in predictive diagnosis of cancerous invasion depth [9], but use of NBIME is preferred in clinical practice due to its technical simplicity and lack of a need for mucosal staining.

We previously reported the technique of NBIME with acetic acid enhancement (A-NBIME) as an alternative modality for CV-MCE [10]. The visualized pit pattern and the diagnostic accuracy of A-NBIME is equivalent to those of CV-MCE ( $\triangleright$ Fig. 1), but the procedure is technically simpler and less time-consuming. Acetic acid reportedly changes the molecular structure of epithelial cellular proteins transiently [11-13], and A-NBIME clearly visualizes the pit pattern; glandular crypts appear deep brown, and the intervening portion appears whitish.

To compare histologic diagnostic accuracy of colonic polyps and the diagnostic reproducibility among WLE, NBIME, and ANBIME, we conducted a retrospective study according to the Standards for the Reporting of Diagnostic Accuracy Studies Initiative [14]. This study initially started as a prospective crosssectional analysis, but the registration period overlapped with the transition period establishing a universal NBI magnifying endoscopic classification for colorectal tumors (Japan NBI Expert Team: JNET classification), which was formally proposed in June 2014, and published in July 2016 [15]. We waited for publication of this new universal classification to start evaluating endoscopic images, leading to a 19-month delay from the completion of patient registration. Therefore, we have treated this study as a retrospective analysis.

\section{Patients and methods}

\section{Participants}

Between February 2010 and November 2014, 718 colorectal polyps from 432 consecutive patients were enrolled in this study at Tottori Municipal Hospital. The protocol was approved by the medical ethics committee of the institution, and written informed consent was obtained from all participants.

Inclusion criteria were as follows: (1) a colorectal polyp suspected to be adenoma or early stage cancer, and hyperplasia difficult to differentiate from neoplasm; (2) patients with good general physical condition who were able to tolerate an endoscopic or surgical procedure; (3) patient provision of written informed consent to undergo a series of examinations and treatment protocols.

\section{Colon preparation}

All patients were prepared for colonoscopy with $150 \mathrm{mg}$ of sodium picosulfate hydrate administered the night before the examination and 2 to $3 \mathrm{~L}$ of polyethylene glycol-electrolyte solution administered on the morning of the examination. Patients received scopolamine butylbromide $(10 \mathrm{mg})$ or glucagon $(.5 \mathrm{mg})$ before colonoscopy to inhibit bowel peristalsis if they had no contraindications to these drugs. 


\section{Endoscopic procedure}

Colorectal polyps of all enrolled patients were photographed with WLE, NBIME, and A-NBIME. First, a lesion was carefully observed, and macroscopic images were photographed with WLE. Second, the vascular and surface pattern visualized with NBIME was photographed. Finally, a lesion was instilled with $1.5 \%$ acetic acid solution through the forceps channel of the endoscope, and the pit pattern of the same area viewed with NBIME was photographed with NBIME under acetic acid-enhanced conditions. When there were multiple areas with different magnification findings for one lesion, the most atypical area was photographed. After endoscopic observation, a lesion was resected endoscopically or surgically.

In this study, all endoscopic photographs were taken by a single expert endoscopist (S.K) who had experience with over 3,000 cases of magnification colonoscopy. Lesions with endoscopic images of poor quality, such as out of magnification focus, covered with adhesive mucus or exudate that could not be removed by water washing, and insufficient acetic acid enhancement, were excluded from the current study.

\section{Endoscopic system}

The instruments used in this study were a magnification videoendoscopic system (PCF-240ZI and PCF-Q260AZI, Olympus Medical Systems Co, Ltd, Tokyo, Japan) and a standard optical videoendoscopic system (EVIS LUCERA SPECTRUM system and EVIS LUCERA ELITE system, Olympus Medical Systems Co, Ltd, Tokyo, Japan). Structural enhancement was set to A5 mode for WLE and B8 mode for NBIME and A-NBIME, respectively.

\section{Endoscopic diagnostic criteria for WLE, NBIME and A-NBIME}

The endoscopic image of each modality was diagnosed as hyperplasia; adenoma including sessile serrated adenoma (SSA), mucosal or submucosal slightly invasive cancer (M-SM1 cancer; invasion depth from muscularis mucosae $<1000 \mu \mathrm{m})$; and submucosal deeply invasive cancer (SM2 cancer; invasion depth $\geq 1000 \mu \mathrm{m}$ ), according to the diagnostic criteria below.

\section{WLE diagnosis}

WLE images were diagnosed according to their macroscopic features ( $\triangleright$ Fig. 2, left), which were characterized by shape, color, and mucosal surface structure of the lesions [16-20]. The diagnostic criterion with WLE for each histologic characteristic was as follows: Hyperplasia was diagnosed as a sessile and smooth-surfaced polyp that was whitish or without discoloration. Adenoma was diagnosed as a variably shaped, smooth or lobed-surfaced polyp, or a sessile and smooth-surfaced polyp covered with much mucus (SSA). Adenocarcinoma was diagnosed as a variably shaped polyp with a granular, focally depressed, or friable surface: SM2 cancer was diagnosed as lesions with a large nodule or loss of lobation, crumbling, fullness, stoke swelling, or fold convergence. Others without such findings were diagnosed as M-SM1 cancer.
NBIME diagnosis

NBIME images were diagnosed according to the JNET classification, which classifies vascular and surface patterns of colonic polyps into four categories (type 1, 2A, 2B, and 3) [15], associated with hyperplasia/SSA, adenoma, M-SM1 cancer, and SM2 cancer, respectively. This classification regards the endoscopic findings of hyperplasia and SSA as the same category. In the current study, a type 1 polyp was differentially diagnosed as hyperplasia or SSA according to recent reports on SSA $[18,21]$

( $>$ Fig. 2, center).

The detailed diagnostic criterion for NBIME for each histologic characteristic was as follows: Type 1 denotes invisible vessels with regular dark or white spots. When a polyp of type 1 showed dilated and branching vessels with an expanded crypt opening, it was diagnosed as SSA. Other type 1 polyps were diagnosed as hyperplasia. A polyp of type $2 \mathrm{~A}$, showing regularly shaped and distributed vessels with a regular tubular, branched, or papillary surface, was diagnosed as adenoma. A polyp of type $2 \mathrm{~B}$, showing irregularly shaped and distributed vessels with an irregular or obscure surface, was diagnosed as M-SM1 cancer. A polyp of type 3 , showing interrupted thick vessels with an amorphous surface, was diagnosed as SM2 cancer.

\section{A-NBIME diagnosis}

A-NBIME images were diagnosed according to pit pattern classification ( $\triangleright$ Fig. 2 , right part) which classifies the crypt opening shape into five categories. Type I, II, III/IV, and V are associated with normal mucosa, hyperplasia/SSA, adenoma, and cancer, respectively [3]. Type $\mathrm{V}$ is subclassified into $\mathrm{V}_{\text {I-L }}$ (irregular, low grade), $V_{I^{-}-H}$ (irregular, high grade), and $V_{N}$ (non-structure); $V_{\text {I-L }}$ and $\mathrm{V}_{\mathrm{I}-\mathrm{H}} / \mathrm{V}_{\mathrm{N}}$ are associated with M-SM1 cancer and SM2 cancer, respectively $[22,23]$. As SSA is included in the same category with hyperplasia, a polyp of type II was differentially diagnosed as hyperplasia or SSA according to additional findings [24].

The diagnostic criterion for A-NBIME for each histologic type was as follows [10]: Type II denotes an asteroid-like pit. When a polyp of type II showed an expanded crypt opening, it was diagnosed as SSA. Others of type II were diagnosed as hyperplasia. A polyp of Type III/IV, showing a regularly formed and distributed crypt opening that is roundish, tubular, dendritic or gyrus-like, was diagnosed as an adenoma. A polyp of type $\mathrm{V}_{\mathrm{I}-\mathrm{L}}$, showing an irregularly formed and distributed crypt opening with clear contours, was diagnosed as M-SM1 cancer. A polyp of type $\mathrm{V}_{\mathrm{I}-\mathrm{H}} / \mathrm{V}_{\mathrm{N}}$, showing a highly destroyed crypt opening or amorphous surface, was diagnosed as SM2 cancer.

\section{Diagnosis of endoscopic images}

Endoscopic images from WLE, NBIME, and A-NBIME were displayed separately for each modality to enable evaluation of its inherent diagnostic accuracy exclusive of the influence of the other modalities. The endoscopic image collection was created after publication of the JNET classification, and reviewed by three expert endoscopists (T.Y., N.I. and K.S.) independently. All of the reviewers had experience reviewing over 3,000 cases of magnifying colonoscopy and who had no prior knowledge of the histologic findings of the lesions. They diagnosed the colo- 

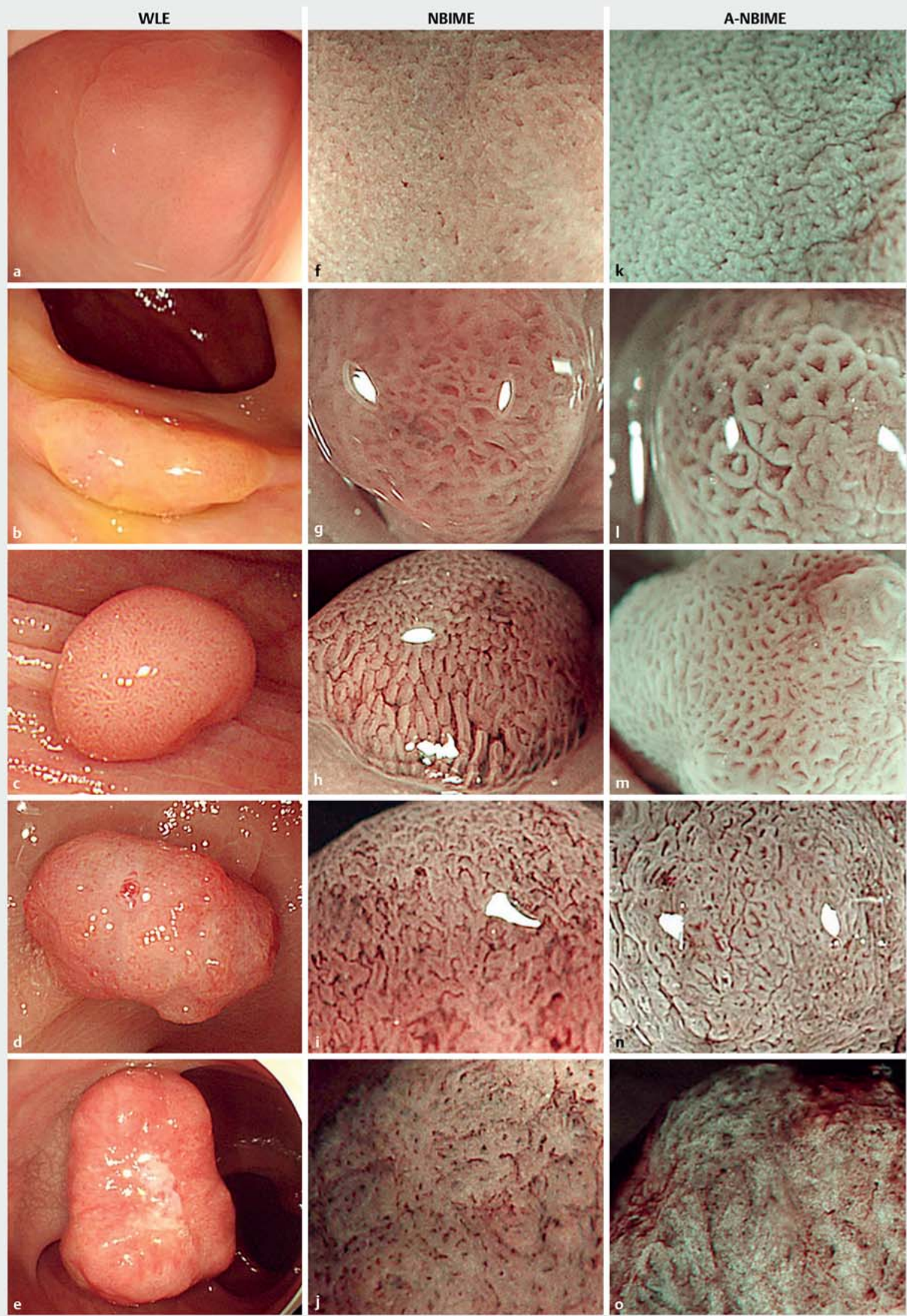

$>$ Fig. 2 
rectal polyps on one to two images of WLE, NBIME, and ANBIME, referring to the atlas of endoscopic photography with written explanations. Endoscopic diagnosis was selected from four choices; hyperplasia, adenoma (including SSA), M-SM1 cancer, and SM2 cancer.

\section{Histologic evaluation}

Histologic diagnosis was treated as the gold standard. A single expert pathologist (K.K), who had no information regarding the endoscopic findings prior to the histologic diagnosis, diagnosed the histologic findings according to the criteria of the Japanese Research Society for Cancer of the Colon and Rectum [25]. When histologic evaluation was difficult due to histologic damage, such lesions were excluded from the analysis.

\section{Diagnostic accuracy}

Diagnostic accuracy was defined as the rate at which a histologic result corresponded with endoscopic diagnosis of hyperplasia, adenoma, M-SM1 cancer, and SM2 cancer. When two or all three of the experts agreed with the endoscopic diagnosis of a lesion, it was regarded as an "experts-agreed" diagnosis when calculating the diagnostic accuracy of the modality. If a lesion received three different diagnoses from the three experts, it was excluded from the study as a "disagreed" diagnosis. Overall diagnostic accuracy and diagnostic sensitivity, specificity, positive predictive value (PPV), and negative predictive value (NPV) for each histological characteristic were compared statistically among WLE, NBIME, and A-NBIME based on the "expertsagreed" diagnosis.

\section{Interobserver diagnostic agreement}

To certify the clinical feasibility of each modality, Fleiss's kappa statistics were calculated as a measure of interobserver diagnostic agreement for the three reviewers and compared statistically among modalities.

\section{Sample size calculation}

In the current study, to calculate the sample size necessary for comparing the diagnostic accuracy of these modalities, the first 100 enrolled lesions, including two hyperplasias, 84 adenomas (including 4 SSA), 12 M-SM1 cancer, and 2 SM2 cancer, were analyzed as a pilot study based on the "expert-agreed" diagnosis. The diagnostic accuracies of WLE, NBIME, and A-NBIME were $80 \%$ (80/100), $78 \%$ (78/100), and $90 \%$ (90/100), respectively, showing a statistical tendency for the diagnostic superiority of A-NBIME to WLE $(P=.047)$ and NBIME $(P=.02)$; significance levels were lowered to 0.016 due to Bonferroni correction for multiple testing. To confirm the superiority of A-NBIME to both WLE and NBIME for diagnostic accuracy with a clinically meaningful difference of $5 \%$, a sample size of 627 lesions would be needed to provide a statistical power of $80 \%$ at an alpha level of $5 \%$ (two-sided tests). Approximately $10 \%$ of the enrolled patients were possibly expected to drop out due to various causes. Consequently, a total of 698 lesions was considered to be needed for enrollment.

The adequate sample size for kappa statistics is still controversial, but a size of 50 items or more reportedly is necessary for calculating interobserver reliability [26]. In this study, the kappa statistics for interobserver diagnostic agreement were analyzed with a complete survey of all enrolled lesions with the sample size for diagnostic accuracy.

\section{Statistical analysis}

Diagnostic accuracy was expressed as a point estimate with an exact $95 \%$ confidence interval $(95 \% \mathrm{Cl})$, and it was compared statistically between A-NBIME and other modalities by the chisquare test with adequate sample size. As the analyses were not multiple testing here, the significance level was set to $P<0.05$.

Interobsever diagnostic agreement was analyzed with Fleiss's kappa statistics, and the results are expressed as point estimates of kappa with $95 \% \mathrm{Cls}$. In theory, perfect disagreement has a kappa value of- 1 , and perfect agreement has a kappa value of +1 . A value of 0 means an agreement by chance alone. According to the Landis and Koch scale, the kappa value was estimated as follows: $0.01-0.2$ slight, $0.21-0.4$ fair, $0.41-$ 0.6 moderate, $0.61-0.8$ substantial and $0.81-1.0$ almost perfect [27].

\section{Results}

A total of 718 colorectal polyps in 432 patients were photographed with WLE, NBIME and A-NBIME and resected endoscopically or surgically. Endoscopic images of 71 polyps were of poor quality: 26 were insufficiently enhanced by acetic acid, and 45 were out of focus. Histologic findings were difficult to diagnose for 12 polyps due to 7 damaged specimens and 5 removal failures. Six polyps were diagnosed as advanced colonic cancers after surgical resection, and one polyp was diagnosed as a leiomyoma after endoscopic resection. These 90 polyps were excluded from the analysis, and a total of 628 polyps were analyzed ( $\triangleright$ Fig. 3 ). Locations of the included polyps were right hemicolon $(n=226)$, left hemicolon $(n=316)$, and rectum $(n=76)$. The median size (range) was $7(2-60) \mathrm{mm}$. On gross appearance, 425 polyps were protruded, 200 were flat, and 3 were depressed types. Histologic characteristics were 38 hyperplasias, 488 adenomas, 72 M-SM1 cancers, and 30 SM2 cancers.

- Fig. 2 Endoscopic classification of colorectal polyps. WLE images (left part) were diagnosed as a hyperplasia, b sessile serrated adenoma or c adenoma, d M-SM1 cancer, and e SM2 cancer, according to the traditional criteria. NBIME images (center part) were diagnosed according to the JNET classification: $\mathbf{f}$ type $1, \mathbf{g}$ type 1 of SSA or $\mathbf{h}$ type $2 \mathrm{~A}, \mathbf{i}$ type $2 \mathrm{~B}$, and $\mathbf{j}$ type 3 , associated with hyperplasia, adenoma, M-SM1 cancer, and SM2 cancer, respectively. A-NBIME images (right) were diagnosed according to the pit pattern classification $\mathbf{k}$ type II, I type II of SSA/m type III or IV, $\mathbf{n}$ type $\mathrm{V}_{1}$-low grade, $\mathbf{o}$ and type $\mathrm{V}_{\mathrm{l}}$-high grade or $\mathrm{V}_{\mathrm{N}}$, associated with hyperplasia, adenoma, M-SM1 cancer, and SM2 cancer, respectively. For a detailed explanation of each classification, refer to the "Patients and methods" section. 
718 colorectal polyps were assessed for enrollment and photographed with WLE, NBIME and A-NBIME

71 images of poor quality

- 26 insufficient for acetic acid enhancement

- 45 out of focus

12 impossible to diagnose histologically

- 7 damaged

- 5 failed to remove

7 met histological exclusion criteria

- 6 advanced colorectal cancers

- 1 leiomyoma derived from muscularis mucosa

628 included in review by 3 expert reviewers

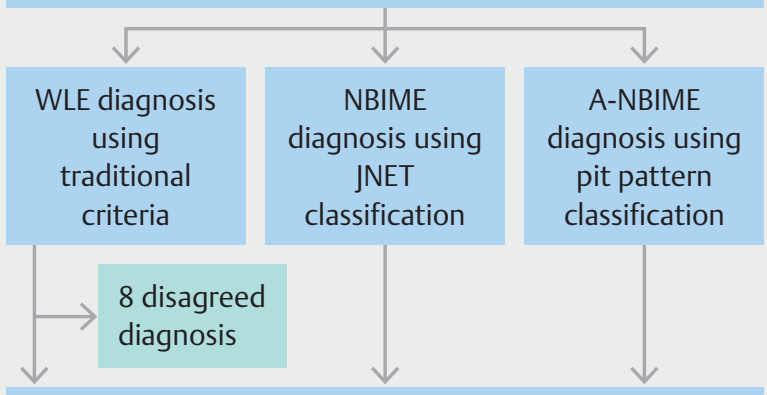

Calculation of diagnostic accuracy by expert-agreed diagnosis

Fig. 3 Consort diagram showing the number of enrolled lesions in this study.

\section{Overall diagnostic accuracy}

The association between the expert-agreed diagnosis and the histologic result with each modality is shown in Table 1 . The WLE diagnosis was "disagreed" for eight lesions (1.3\%), but the NBIME and A-NBIME diagnoses were agreed upon by two or all three reviewers for all 628 lesions. Diagnostic accuracy $(95 \% \mathrm{Cl}$; $\mathrm{n} / \mathrm{N})$ of WLE, NBIME, and A-NBIME was $80.8 \%$ (77.4\%-83.8\%; $501 / 620), \quad 79.3 \%(75.9 \%-82.4 \% ; 498 / 628)$, and $86.1 \%$ (83.2\%-88.7\%; 541/628), respectively. A-NBIME showed a statistically significantly higher rate of diagnostic accuracy compared with WLE and NBIME $(P<.016)$, and there was no statistically significant difference between WLE and NBIME.

\section{Association between WLE diagnosis and individual histologic characteristics}

The association between the WLE diagnosis and the histologic results is shown at the top of $\nabla$ Table $2 a$. Diagnostic sensitivity, specificity, PPV, and NPV with $95 \% \mathrm{Cl}$ for each histologic characteristic was $29.7 \%$ (15.8\%-46.9\%), 98.2\% (96.8\%-99.1\%), $52.4 \%$ (29.7\%-74.2\%), and 95.6\% (93.7\%-97.1\%), respectively, for hyperplasia; $90.0 \%$ (87.0\%-92.5\%), 61.3\% (52.6\%$69.5 \%$ ), $89.1 \%$ (86.0\%-91.7\%), and 63.6\% (54.8\%-71.8\%),
- Table 1 Diagnostic accuracy of the 'experts-agreed' diagnosis of each modality.

\begin{tabular}{|c|c|}
\hline Modality & Diagnostic accuracy (95\% Cl) \\
\hline WLE & $80.8 \%(77.4 \%-83.8 \%)^{1,2}$ \\
\hline NBIME & $79.3 \%(75.9 \%-82.4 \%)^{1,3}$ \\
\hline A-NBIME & $86.1 \%(83.2 \%-88.7 \%)^{3,2}$ \\
\hline \multicolumn{2}{|c|}{$\begin{array}{l}\text { WLE, white light endoscopy; NBIME, magnification endoscopy with narrow- } \\
\text { band imaging; A-NBIME, magnification endoscopy with acetic acid en- } \\
\text { hanced narrow-band imaging; } \mathrm{CI} \text {, confidence interval. } \\
{ }^{1} P=0.75 \text {; WLE vs. NBIME } \\
{ }^{2} P<0.016 \text {; WLE vs. A-NBIME } \\
{ }^{3} P<0.016 \text {; NBIME vs. A-NBIME }\end{array}$} \\
\hline
\end{tabular}

respectively, for adenoma; 58.5\% (46.1\%-70.2\%), $90.3 \%$ (86.0\%-91.7\%), 43.6\% (33.4\%-54.2\%), and 94.4\% (92.1\%$96.2 \%)$, respectively, for M-SM1 cancer; and $48.2 \%$ (29.2\%67.4\%), 99.4\% (98.5\%-99.9\%), 82.4\% (56.3\%-96.2\%), and $97.5 \%$ (95.9\%-98.6\%), respectively, for SM2 cancer.

\section{Association between NBIME diagnosis and individual histologic characteristics}

The association between the NBIME diagnosis and the histologic results is shown in the middle of $\mathbf{r}$ Table $\mathbf{2 b}$. Diagnostic sensitivity, specificity, PPV, and NPV with $95 \% \mathrm{CI}$ for each histologic characteristic was $28.9 \%(15.4 \%-45.9 \%)$, $99.3 \%(98.2 \%-$ 99.8\%), $73.3 \%$ (44.9\%-92.2\%), and 95.5\% (93.6\%-97.0\%), respectively, for hyperplasia; $85.0 \%$ (81.5\%-88.0\%), $65.7 \%$ (57.2\%-73.5\%), 89.6\% (86.4\%-92.2\%), and $55.7 \%(47.8 \%-$ $63.4 \%)$, respectively, for adenoma; 69.4\% (57.4\%-79.7\%), $86.3 \%(83.1 \%-89.0 \%), \quad 39.7 \%(31.0 \%-48.7 \%)$, and $95.6 \%$ (93.4\%-97.2\%), respectively, for M-SM1 cancer; and $73.3 \%$ (54.1\%-87.7\%), 99.6\% (98.8\%-99.9\%), 91.6\% (73.0\%-98.9\%), and $98.6 \%$ (97.4\%-99.4\%), respectively, for SM2 cancer.

\section{Association between A-NBIME diagnosis and individual histologic characteristics}

A-NBIME enabled clear visualization of the pit pattern in $96.3 \%$ (692/718) of enrolled polyps, showing a high feasibility. The association between the A-NBIME diagnosis and the histologic results is shown at the bottom of $\mathbf{r a b l e 2 c}$. Diagnostic sensitivity, specificity, PPV, and NPV with $95 \% \mathrm{Cl}$ for each histologic characteristic was $55.2 \%$ (38.3\%-71.3\%), 98.6\% (97.3\%-99.4 $\%$ ), $72.4 \%(52.7 \%-87.2 \%)$, and $97.1 \%$ (95.4\%-98.3\%), respectively, for hyperplasia; $94.4 \%$ (92.0\%-96.3\%), 65.0\% (56.4 \%-72.8\%), 90.4\% (87.5\%-92.8\%), and $77.1 \%$ (68.4\%-84.3\%), respectively, for adenoma; $54.1 \%$ (42.0\%-65.9\%), $94.7 \%$ (92.5\%-96.4\%), 57.4\% (44.7\%-69.2\%), and $94.1 \%(91.8 \%-$ $95.9 \%)$, respectively, for M-SM1 cancer; and 63.3\% (43.8\%$80.0 \%$ ), 99.6\% (98.8\%-99.9\%), 90.4\% (69.6\%-98.8\%), and $98.1 \%(96.7 \%-99.0 \%)$, respectively, for SM2 cancer. 
- Table 2a Association between the experts-agreed WLE diagnosis and histologic characteristics.

\begin{tabular}{|l|l|l|l|l|}
\hline \multicolumn{2}{|l|}{ WLE (n=620) } & \multicolumn{4}{l|}{ Histologic characteristics } \\
\hline WLE diagnosis & Hyperplasia & Adenoma & M-SM1 cancer & SM2 cancer \\
\hline & $\mathbf{n = 3 7}$ & $\mathbf{n = 4 8 3}$ & $\mathbf{n}=\mathbf{7 0}$ & $\mathbf{n = 3 0}$ \\
\hline Hyperplasia $n(\%)$ & $11(52.4)$ & $10(47.6)$ & 0 & 0 \\
\hline Adenoma $n(\%)$ & $26(5.3)$ & $435(89.1)$ & $26(5.3)$ & $1(0.2)$ \\
\hline M-SM1 cancer $n(\%)$ & 0 & $38(40.4)$ & $41(43.6)$ & $15(16.0)$ \\
\hline SM2 cancer $n(\%)$ & 0 & 0 & $3(17.6)$ & $14(82.4)$ \\
\hline
\end{tabular}

- Table 2b Association between the experts-agreed NBIME diagnosis and histologic characteristics.

\section{NBIME $(n=628)$}

\begin{tabular}{|l|l|l|l|l|}
\hline & & Histologic characteristics & SM2 cancer \\
\hline NBIME diagnosis & Hyperplasia & Adenoma & M-SM1 cancer & $\mathbf{n = 3 0}$ \\
\hline Hyperplasia $n(\%)$ & $\mathbf{n = 3 8}$ & $\mathbf{n = 4 8 8}$ & $\mathbf{n = 7 2}$ & 0 \\
\hline Adenoma $\mathrm{n}(\%)$ & $11(73.3)$ & $4(26.7)$ & 0 & $1(0.2)$ \\
\hline M-SM1 cancer $\mathrm{n}(\%)$ & $26(5.6)$ & $415(89.6)$ & $21(4.5)$ & $7(5.6)$ \\
\hline SM2 cancer $\mathrm{n}(\%)$ & $1(0.7)$ & $68(54.0)$ & $50(39.7)$ & $1(4.2)$ \\
\hline
\end{tabular}

- Table 2c Association between the experts-agreed A-NBIME diagnosis and histologic characteristics.

\begin{tabular}{|c|c|c|c|c|}
\hline \multicolumn{5}{|l|}{ A-NBIME $(n=628)$} \\
\hline \multirow[b]{2}{*}{ NBIME diagnosis } & \multirow[b]{2}{*}{ Hyperplasia } & \multicolumn{3}{|c|}{ Histologic characteristics } \\
\hline & & Adenoma & M-SM1 cancer & SM2 cancer \\
\hline & $n=38$ & $n=488$ & $n=72$ & $\mathrm{n}=\mathbf{3 0}$ \\
\hline Hyperplasia n (\%) & $21(72.4)$ & $6(20.6)$ & $2(7.0)$ & 0 \\
\hline Adenoma n (\%) & $17(3.3)$ & $461(90.4)$ & $30(5.9)$ & $2(0.4)$ \\
\hline M-SM1 cancer n (\%) & 0 & $20(29.4)$ & $39(57.4)$ & $9(13.2)$ \\
\hline SM2 cancer n (\%) & 0 & $1(4.8)$ & $1(4.8)$ & $19(90.4)$ \\
\hline
\end{tabular}

\section{Interobserver diagnostic agreement}

Kappa values $(95 \% \mathrm{Cl})$ for interobserver diagnostic agreement of WLE, NBIME, and A-NBIME were 0.43 (0.39-0.46), 0.52 (0.49-0.56), and $0.65(0.62-0.69)$, respectively, showing "fair" to "moderate" agreement for WLE, "moderate" agreement for NBIME, and "substantial" agreement for A-NBIME. WLE showed insufficient diagnostic agreement, even among expert reviewers, which was statistically significantly lower than those of NBIME and A-NBIME. In contrast, A-NBIME showed the statistically significantly highest agreement among modalities ( $\triangleright$ Table 3$)$.

\section{Discussion}

Magnification colonoscopy has been reported to provide higher diagnostic accuracy than WLE for colorectal polyps, but most reports have suggested a differential diagnostic ability between neoplastic and non-neoplastic polyps [28-30]. In addition, the clinical feasibility, such as diagnostic reproducibility, has not been fully discussed to date. We performed this retro- 
- Table 3 Fleiss's kappa statistics of the interobserver agreement of the WLE, NBIME, and A-NBIME diagnosis among the three expert reviewers.

\begin{tabular}{|l|l|}
\hline Modality & Fleiss's kappa value $\mathbf{( 9 5 \% \mathbf { C I } )}$ \\
\hline WLE & $0.43(0.39-0.46)$ \\
\hline NBIME & $0.52(0.49-0.56)$ \\
\hline A-NBIME & $0.65(0.62-0.69)$ \\
\hline $\begin{array}{l}\text { WLE, white light endoscopy; NBIME, magnification endoscopy with narrow- } \\
\text { band imaging; A-NBIME, magnification endoscopy with acetic acid-en- } \\
\text { hanced narrow-band imaging; Cl, confidence interval }\end{array}$ \\
\hline
\end{tabular}

spective study to compare diagnostic accuracy of more detailed histologic characteristics and interobserver diagnostic agreement among modalities. Acetic acid is an amphipathic molecular with small molecular weight, while crystal violet is a hydrophobic one with a large molecular weight. According to Fick's law, acetic acid passes through the cell wall covered with mucus to cause mucosal discoloration more rapidly than crystal violet [31], leading to the short procedure time. In addition, the pit pattern with A-NBIME is clearly visualized equivalent to that with CV-MCE [10].
In the current study, the specificity $(95 \% \mathrm{Cl})$ of hyperplasia and SM2 cancer with WLE were 98.2\% (96.8\%-99.1\%) and $99.4 \%$ (98.5\%-99.9\%), showing sufficiently high accuracy to establish an indication for endoscopic resection without magnifying observation. In addition, the overall diagnostic accuracy $(95 \% \mathrm{Cl})$ of WLE was good, at $80.8 \%(77.4 \%-83.8 \%)$. This good diagnostic ability of WLE may be due largely to the high expertise of the reviewers, but it may also suggest that WLE without magnifying observation can be permitted in clinical endoscopic practice for colorectal polyps.

Overall diagnostic accuracy was statistically significantly higher with A-NBIME than with WLE and NBIME. This result is theoretically easy to understand, considering that the pit pattern visualized with A-NBIME is directly linked to the glandular structural atypia of the colorectal polyps. However, the actual difference was clinically small, at approximately 5\%. NBIME had a lower PPV for M-SM1 cancer $(P<.05)$ as did WLE $(P=.08)$ compared with $A-N B I M E$, resulting in its inferiority to $A-N B I M E$ in overall diagnostic accuracy. JNET classification type 2B included many adenomas, accounting for the majority of the enrolled polyps. An adenoma of type $2 \mathrm{~B}$ with an obscure surface pattern is difficult to diagnose by NBIME, suggesting that the vessel pattern does not necessarily reflect the glandular structural atypia ( $>$ Fig.4). The PPV for hyperplasia was low for all
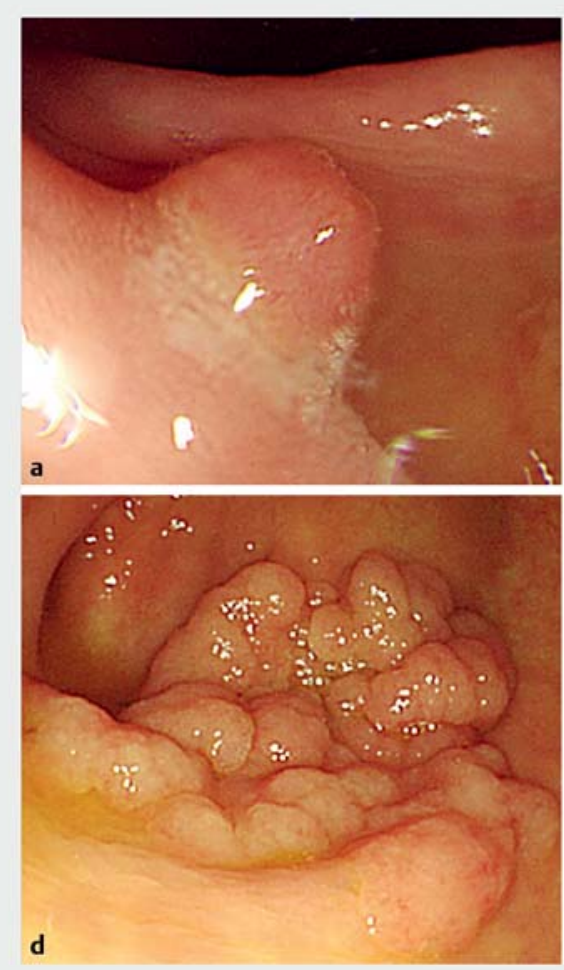
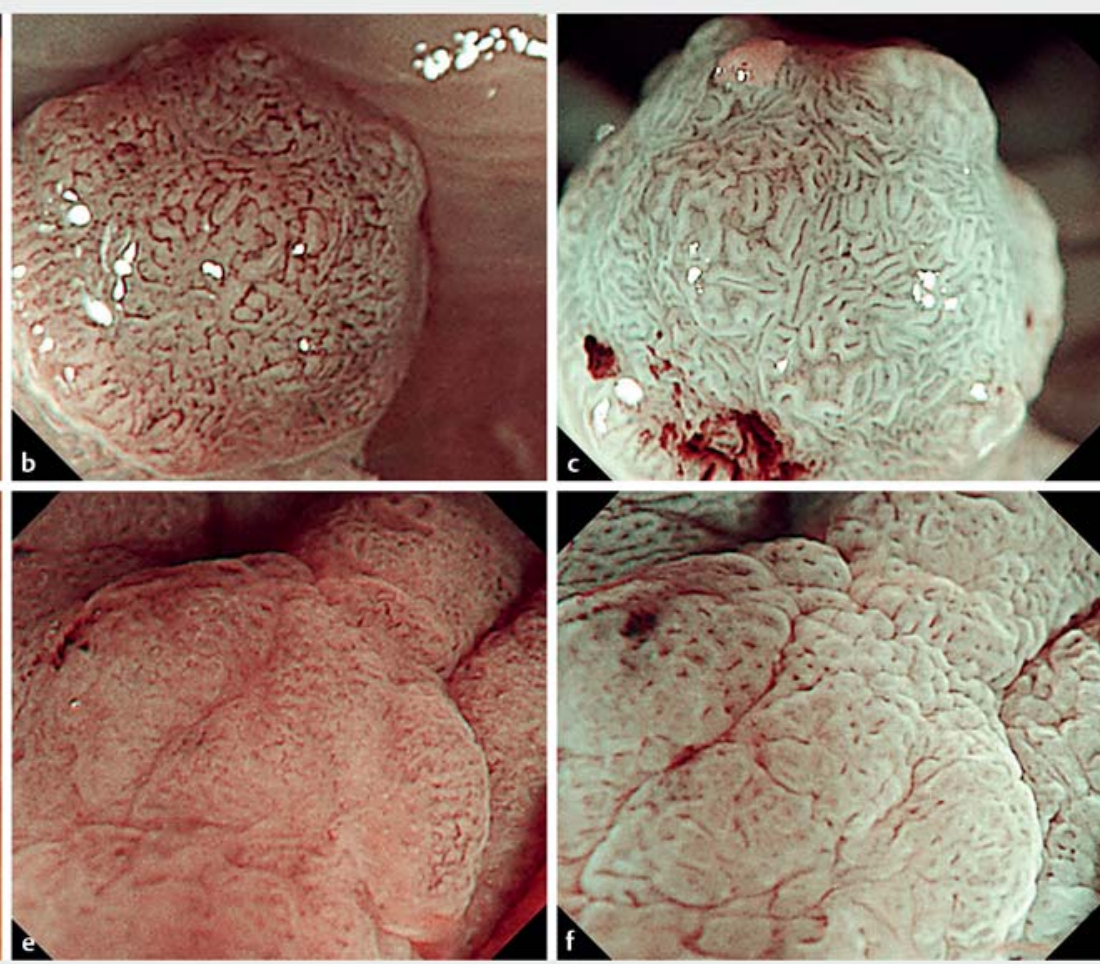

- Fig. 4 Diagnostic discrepancy among modalities. a Case 1 with adenoma: WLE shows a reddish and roundish polyp, suggesting adenoma. b However, NBIME shows an irregularly shaped and dilated vessel with an obscure surface, type 2B in the JNET classification, suggesting M-SM1 cancer. c A-NBIME shows a regularly formed and distributed tubular crypt opening, a type III pit pattern, suggesting adenoma. $\mathbf{d}$ Case 2 with adenoma: WLE shows a laterally spreading tumor with an irregular surface, suggesting M-SM1 cancer. e NBIME shows an obscure vessel with an irregular surface, type 2B in the JNET classification, suggesting M-SM1 cancer. $\mathbf{f}$ However, A-NBIME shows a regularly distributed roundish or tubular or crypt opening, a type III pit pattern, suggesting adenoma. 
modalities, although it was slightly higher with magnification endoscopy than with WLE. A visually evident hyperplasia is not resected in this study. This selection bias may cause the enrollment of hyperplasia that is difficult to differentiate from neoplastic polyps. On the other hand, the PPV for adenoma and SM2 cancer was sufficiently good for all three modalities. Generally, as the proportion of adenoma increases, the overall diagnostic accuracy is pulled upward. We must carefully interpret diagnostic accuracy in consideration of the correlation between PPV and histologic proportion. We consider WLE should be followed by NBIME for diagnosis of a colorectal polyp, unless it is diagnosed as an adenoma by WLE with strong confidence. In addition, a polyp suspected on NBIME to be M-SM1 cancer should be estimated by the pit pattern analysis, although the PPV for M-SM1 cancer with A-NBIME was also insufficient in the current study.

WLE demonstrated insufficient diagnostic reproducibility, even among experts. NBIME, A-NBIME showed acceptable reproducibility, and A-NBIME showed the highest reproducibility of the three modalities. When the reviewers diagnose the colorectal polyps, they analyze the various types of visual information, including the size, shape, color, and mucosal surface with WLE, the vessel and surface pattern with NBIME, and the pit pattern with A-NBIME. While the WLE diagnosis requires a comprehensive analysis of multiple factors, magnifying diagnosis using the classification system is a simplified diagnostic process. Especially with the A-NBIME diagnosis, the reviewers have only to judge the shape of the crypt opening, leading to a high diagnostic agreement.

The current study has some limitations. First, the diagnostic process used was not real-time diagnosis. That may have caused selection bias on image presentation or misdiagnosis due to a lack of information. A multicenter, prospective, real-time comparative study among modalities is now ongoing to estimate the actual value of advanced imaging (UMIN000034549). Previous systematic reviews suggested the clinical significance of advanced imaging, but its diagnostic superiority to WLE has not yet been confirmed [32-34]. Second, we modified classifications of magnification endoscopy to distinguish SSA from hyperplasia. The diagnostic accuracy in this study is not strictly the same as that with the original version of JNET and pit pattern classification. Third, a single expert pathologist was in charge of histopathological diagnosis in this study. Last, repeated use of acetic acid for A-NBIME sometimes induces colonic peristalsis, requiring an additional spasmolysis. Adequate dosage in an examination should be estimated in future study.

\section{Conclusion}

In conclusion, WLE showed high accuracy for endoscopic resection in expert diagnosis, but its diagnostic reproducibility was insufficient compared with NBIME and A-NBIME. Among the three modalities, A-NBIME demonstrated possible superiority in diagnostic accuracy and reproducibility in histologic predictive diagnosis of colorectal polyps.

\section{Competing interests}

The authors declare that they have no conflict of interest.

References

[1] IARC. Cancer Today.http://gco.iarc.fr/today/fact-sheets-cancers

[2] Winawer S], Zauber AG, Ho MN et al. Prevention of colorectal cancer by colonoscopic polypectomy: The National Polyp Study Workgroup. N Engl J Med 1993; 329: 1977-1981

[3] Kudo S, Tamura S, Nakajima T et al. Diagnosis of colorectal tumorous lesions by magnifying endoscopy. Gastrointest Endosc 1996; 44: 814

[4] Fujii T, Hasegawa RT, Saitoh Y et al. Chromoscopy during colonoscopy. Endoscopy 2001; 33: 1036-1041

[5] Tanaka S, Kaltenbach T, Chayama K et al. High magnification colonoscopy (with videos). Gastrointest Endosc 2006; 64: 604-613

[6] Machida $\mathrm{H}$, Sano $\mathrm{Y}$, Hamamoto $\mathrm{Y}$ et al. Narrow-band imaging in the diagnosis of colorectal mucosal lesions a pilot study. Endoscopy 2004; 36: 1094-1098

[7] Kanao H, Tanaka S, Oka S et al. Narrow-band imaging magnification predicts the histology and invasion depth of colorectal tumors. Gastrointest Endosc 2009; 69: 631-636

[8] Minoda Y, Ogino H, Chinen T et al. The objective validity of the JNET classification system for the differential diagnosis of colorectal polyps. Dig Endosc 2019; 31: 544-551

[9] Wada Y, Kashida H, Kudo S et al. Diagnostic accuracy of pit pattern and vascular pattern analysis in colorectal lesions. Dig Endosc 2010; 22: 192-199

[10] Shibagaki K, Amano Y, Ishimura N et al. Magnification endoscopy with acetic acid- enhancement and a narrow-band imaging system for pit pattern diagnosis of colorectal neoplasms. J Clin Gastroenterol 2015; 49: 306-312

[11] Yagi K, Aruga Y, Nakamura A et al. The study of dynamic magnifying endoscopy in gastric neoplasia. Gastrointest Endosc 2005; 62: 963969

[12] Togashi K, Hewett DG, Whitaker DA et al. The use of acetic acid in magnification chromocolonoscopy for pit pattern analysis of small polyps. Endoscopy 2006; 38: 613-616

[13] Goto N, Kusaka T, Tanaka H et al. Magnifying narrow-band imaging with acetic acid to diagnose early colorectal cancer. World J Gastroenterol 2014; 20: 16306-16310

[14] Bossuyt PM, Reitsma JB, Bruns DE et al. The STARD statement for reporting studies of diagnostic accuracy: explanation and elaboration. Ann Intern Med 2003; 138: W1-W12

[15] Sano Y, Tanaka S, Kudo SE et al. Narrow-band imaging (NBI) magnifying endoscopic classification of colorectal tumors proposed by the Japan NBI Expert Team. Dig Endosc 2016; 28: 526-533

[16] Eberl T. Polyps and polyposis syndromes. In: Helmut M (eds) Atlas of Colonoscopy. New York: Georg Thieme Verlag; 2006: 66-80

[17] Hazewinkel Y, Ceron LM, East E] et al. Endoscopic features of sessile serrated adenomas: validation by international experts using highresolution white-light endoscopy and narrow-band imaging. Gastrointest Endosc 2013; 77: 916-924

[18] Uraoka T, Higashi R, Horii J et al. Prospective evaluation of endoscopic criteria characteristic of sessile serrated adenoma/polyp. J Gastroenterol 2015; 50: 555-563

[19] Uraoka T, Saito Y, Matsuda T et al. Endoscopic indication for endoscopic mucosal resection of laterally spreading tumor in the colorectum. Gut 2006; 55: 1592-1597 
[20] Ikehara H, Saito Y, Matsuda T et al. Diagnosis of depth of invasion for early colorectal cancer using magnifying colonoscopy. J Gastroenterol Hepatol 2010; 25: 905-912

[21] Yamada M, Sakamoto T, Otake Y et al. Investigating endoscopic features of sessile serrated adenomas/polyps by using narrow-band imaging with optical magnification. Gastrointest Endosc 2015; 82: 108-117

[22] Kashida H, Kudo S. Magnifying colonoscopy, early colorectal cancer and flat adenomas. In: Waye JD, Rex DK, Williams CB (eds) Colonoscopy; Principles and Practice. 2nd ed. Malden, USA, Oxford, UK, Melbourne, Australia: Blackwell; 2009: 412-422

[23] Kudo S, Miyachi H, Endo S. Magnifying chromocolonoscopy and tattooing. In: Classen M, Tytgat GNJ, Lightdale CJ (eds) Gastroenterol Endosc. 2nd edn. New York, USA: Thieme; 2010: 197-202

[24] Kimura T, Yamamoto E, Yamano $\mathrm{H}$ et al. A novel pit pattern identifies the precursor of colorectal cancer derived from sessile serrated adenoma. Am J Gastroenterol 2012; 107: 460-469

[25] Japanese Society for Cancer of the Colon and Rectum. General rules for clinical and pathological studies on cancer of the colon, rectum and anus [Japanese]. 8th ed. Tokyo: Kanehara; 2013

[26] Lacy S, Riffe D. Sampling error and selecting intercoder reliability sample for normal content categories. Journal Mass Commun Q 1996; 73: 963-973

[27] Landis JR, Koch GG. The measurement of observer agreement for categorical data. Biometrics 1977; 33: 159-174
[28] Tung SY 1, Wu CS, Su MY. Magnifying colonoscopy in differentiating neoplastic from nonneoplastic colorectal lesions. Am J Gastroenterol 2001; 96: 2628-2632

[29] Konishi K, Kaneko K, Kurahashi T et al. A comparison of magnifying and nonmagnifying colonoscopy for diagnosis of colorectal polyps: a prospective study. Gastrointest Endosc 2003; 57: 48-53

[30] Chiu HM, Chang CY, Chen CC et al. A prospective comparative study of narrow-band imaging, chromoendoscopy, and conventional colonoscopy in the diagnosis of colorectal neoplasia. Gut 2007; 56: $373-$ 379

[31] Smith WF. Foundations of Materials Science and Engineering. 3rd ed. McGraw-Hill; 2004

[32] Wanders LK, East JE, Uitentuis SE et al. Diagnostic performance of narrowed spectrum endoscopy, autofluorescence imaging, and confocal laser endomicroscopy for optical diagnosis of colonic polyps: a meta-analysis. Lancet Oncol 2013; 14: 1337-1347

[33] Kamiński MF, Hassan C, Bisschops R et al. Advanced imaging for detection and differentiation of colorectal neoplasia: European Society of Gastrointestinal Endoscopy (ESGE) Guideline. Endoscopy 2014; 46: 435-449

[34] Abu Dayyeh BK, Thosani N, Konda V et al. ASGE Technology Committee systematic review and meta-analysis assessing the ASGE PIVI thresholds for adopting real-time endoscopic assessment of the histology of diminutive colorectal polyps. Gastrointest Endosc 2015; 81 : 502.e1-502.e16 